\title{
Improved Astaxanthin Production of Xanthophyllomyces dendrorhous with Carotenogenesis Stimulating Factors
}

\author{
Bao-Jun Xu', Chang-Tian Li, Eun-Kyoung $\mathrm{Mo}^{2}$ and Chang-Keun Sung*
}

Department of Food Science and Technology, College of Agriculture and Biotechnology, Chungnam National University, Taejon 305-764, Korea ${ }^{1}$ The Pharmaceutical Institute, Dalian University, Dalian 116622, China.

${ }^{2}$ Biotechnology Research Institute, Dbio Inc., Taejon 305-764, Korea.

Received February 16, 2004 / Accepted May 15, 2004

To improve biomass and astaxanthin production by wild-type Xanthophyllomyces dendrorhous simultaneously in shake flask culture, physical factors, nutritional factors and carotenogenesis stimulating factors affecting astaxanthin production were studied on base of HPLC quantitative analysis. The results suggested that carotenogenesis precursor composition acetic acid, mevalonic acid, tomato extract, and carrot extract could increase the productivity of astaxanthin markedly based on the optimized temperature, initial $\mathrm{pH}$ value, carbon and nitrogen sources conditions.

Key words - Astaxanthin, Biomass, Carotenoid, Xanthophyllomyces dendrorhous

Astaxanthin, as a powerful antioxidative reagent that may delay aging and degenerative diseases in human and animals [14], has attracted tremendous commercial interest. It has been used as a pigmentation source for marine fish aquaculture and is also being investigated for food, cosmetic and medical application due to its strong antioxidant activity. Many studies suggested that it has about 10-fold higher antioxidant activity than other carotenoids and has 100-500 times higher activity than a-tocopherol [11,12,15]. The biotechnological process for astaxanthin production is an alternative to the chemical synthesis, owing to high market price and growing demand. The biotechnological production of astaxanthin can be based on the utilization of microalgae, bacteria or yeast. The red yeast, Xanthophyllomyces dendrorhous (formerly known as Phaffia rhodozyma), is one of the most promising microorganisms for the commercial production of astaxanthin [1]. However, the production levels of astaxanthin are rather low, meanwhile increased biomass is usually accompanied by decreased astaxanthin production. For large-scale production of astaxanthin, biomass needs to be maximally produced. However, increased biomass is usually accompanied by decreased carotenoids and astaxanthin production [2]. This study was carried out to find medium components that increase biomass, without decreasing carotenoids and astaxanthin production.

In order to offer theoretical base for commercial

*Corresponding author

Tel : +82-42-821-6722, Fax : +82-42-822-2287

E-mail : kchsung@cnu.ac.kr production of astaxanthin, we investigated optimum physical factors (temperature, initial $\mathrm{pH}$ ), nutritional factors (carbon and nitrogen sources) and carotenogenesis stimulating factors (mevalonic acid, acetic acid, citric acid etc.), affecting astaxanthin production by $X$. dendrorhous in this study.

\section{Materials and Methods}

\section{Chemicals and materials}

Authentic astaxanthin was purchased from Sigma (A9335, St. Louis, MO, USA). All solvents used in chromatography were of HPLC grade (J.T. Baker, NJ, USA). All the yeast samples were obtained from our laboratory by flask culture. Yeast extract, malt extract, beef extract, peptone, glucose, agar were purchased from Difco (Difco Co. Detroit, MI, USA). Tomato extract and carrot extract were obtained by lyophilized $75 \%$ ethanol extract of tomato and carrot.

\section{Yeasts and culture conditions}

For the seed preparation and preservation, the plate culture was performed in incubator at $21^{\circ} \mathrm{C}$. The red yeast $X$. dendrorhous ATCC 24228 was grown on YM media with the following composition: yeast extract, $3 \mathrm{~g} / \mathrm{l} ;$ malt extract, $3 \mathrm{~g} / \mathrm{l}$; peptone, $5 \mathrm{~g} / \mathrm{l}$; glucose, $10 \mathrm{~g} / \mathrm{l}$; agar, $15 \mathrm{~g} / \mathrm{l}$. For the experiments, $X$. dendrorhous was grown on the basal medium PDB containing PDB $24 \mathrm{~g} / 1$. For comparison, other media were utilized to optimize medium: PDBY, PDB $24 \mathrm{~g} / 1$ and yeast $5 \mathrm{~g} / \mathrm{l}$; NA, peptone $5 \mathrm{~g} / \mathrm{l}$, beef extract $3 \mathrm{~g} / 1$ and peptone $5 \mathrm{~g} / \mathrm{l}$; 323Malt, malt extracts 30 
$\mathrm{g} / \mathrm{l}$; YEPD, glucose $20 \mathrm{~g} / \mathrm{l}$, peptone $20 \mathrm{~g} / 1$ and yeast 10 $\mathrm{g} / 1$. All flask cultures were carried out in $250 \mathrm{ml}$ Erlenmeyer flasks containing $100 \mathrm{ml}$ liquid medium, every culture was performed at three parallel level.

\section{Preparation of samples}

Samples were harvested from the flasks after 30 days fermentation and centrifuged. The pellets were washed twice with sterile water and used to measure both biomass (as dry cell weight) and carotenoids. The washed pellets were freezed to dryness by freeze-dryer.

For the analysis of astaxanthin, the samples of $50 \mathrm{mg}$ red yeast lyophilized powder were extracted with $1 \mathrm{ml}$ of dimethylsulphoxide (DMSO) /acetone $(1: 1, \mathrm{v} / \mathrm{v})$ for five times by ultrasonic power at $20^{\circ} \mathrm{C}$ for $20 \mathrm{~min}$. The 5 times extraction solutions were combined and made it up to 5 $\mathrm{ml}$ with extracting solvent. The filtrated solutions were used for HPLC analysis. All manipulations were performed in low light and temperatures, as carotenoids are very sensitive to light, oxygen and heat. Each abovementioned treatment was repeated up to 3 times.

For the measurement of total carotenoid, approximately $25 \mathrm{mg}$ lyophilized culture powder, $5 \mathrm{ml}$ DMSO was added to the centrifuge tubes, vortex for several times with glass bead, then centrifuged. The supernatant were collected in the $10 \mathrm{ml}$ volumetric flask. $2 \mathrm{ml}$ acetone was added to precipitate, suspended vigorously, then centrifuged, the supernatant was added to the volumetric flask. After all the supernatant was collected in the volumetric flask, volume to $10 \mathrm{ml}$ with acetone, and stored for carotenoid analysis.

\section{Instruments and analytical conditions}

The identification and quantification of astaxanthin were carried out by HPLC equipped with two Prostar 210/215 Solvent Delivery Modules and one Prostar 320 UV/VIS detector (Varian Analytical Instruments, Polo Alto, CA, USA). The extract was analyzed (20 $\mu$ l aliquots) using a 250 $\times 4.6 \mathrm{~mm} \mathrm{C}_{18}(5 \mu \mathrm{m})$ column (Ultrasphere, Beckman, U.S.A). The mobile phase consisted of methanol (82\%), dichloromethane $(6.5 \%)$, acetonitrile $(7.5 \%)$ and water $(4.0 \%)$ by volume. The flow-rate was $0.8 \mathrm{ml} / \mathrm{min}$. The detection was monitored at $482 \mathrm{~nm}$. Run time was set between 30 to 45 $\min$.

The quantification of total carotenoids was carried out by UV-Visible spectrophotometer (Varian Cary 100 Conc system, Varian, Australia), read the maximum absorbency at $475 \mathrm{~nm}$ against an acetone blank. The total carotenoids were quantified as the calculated formula: carotenoids $(\mathrm{mg})$ extracted $=A b s_{\max } * 10 \mathrm{ml}$ *dilution factor $/ 250$.

\section{Results and Discussion}

\section{Effect of physical factors on astaxanthin productivity}

Several papers were published on the study of the physical factors ( $\mathrm{pH}$ and temperature) and nutritional factors (carbon and nitrogen sources) of affecting astaxanthin production $[6,9,10]$ by using various wild-type strains and their mutant strains, as well as performing on various nutritional media, various physical factors were reported. Present research was carried out to investigate optimum temperature (Table 1) and initial $\mathrm{pH}$ value (Table 2) of astaxanthin production by wild-type $X$. dendrorhous (ATCC 24228) on basal nutritional medium PDB.

The results (Table 1) showed that temperature range of

Table 1. Effect of temperature on biomass and astaxnthin yield on basal medium PDB

\begin{tabular}{|c|c|c|c|}
\hline Temperature & $\begin{array}{c}\text { Biomass } \\
\text { (dry yeast } \\
\text { g / /) }\end{array}$ & $\begin{array}{l}\text { Astaxanthin } \\
\text { (mg/g yeast) }\end{array}$ & $\begin{array}{c}\text { Astaxanthin } \\
\text { productivity } \\
(\mathrm{mg} / \mathrm{l})\end{array}$ \\
\hline $18^{\circ} \mathrm{C}$ & $1.066 \pm 0.023$ & $0.4933 \pm 0.031$ & $0.5258 \pm 0.045$ \\
\hline $21^{\circ} \mathrm{C}$ & $2.125 \pm 0.089$ & $0.8496 \pm 0.038$ & $1.8054 \pm 0.020$ \\
\hline $23^{\circ} \mathrm{C}$ & $1.784 \pm 0.123$ & $0.5723 \pm 0.056$ & $1.0209 \pm 0.110$ \\
\hline $26^{\circ} \mathrm{C}$ & N.D. ${ }^{a}$ & N.D. ${ }^{a}$ & N.D. ${ }^{\mathrm{a}}$ \\
\hline $28^{\circ} \mathrm{C}$ & N.D. ${ }^{a}$ & N.D. ${ }^{a}$ & N.D. ${ }^{a}$ \\
\hline $30^{\circ} \mathrm{C}$ & N.D. ${ }^{a}$ & N.D. ${ }^{a}$ & N.D. ${ }^{a}$ \\
\hline $32^{\circ} \mathrm{C}$ & N.D. ${ }^{a}$ & N.D. ${ }^{a}$ & N.D. ${ }^{a}$ \\
\hline
\end{tabular}

The results were presented as mean values \pm S.E. of three replicates.

${ }^{a}$ N.D.: not detected.

Table 2. Effect of initial $\mathrm{pH}$ on biomass and astaxanthin yield on basal medium PDB

\begin{tabular}{cccc}
\hline $\begin{array}{c}\text { Initial } \mathrm{pH} \\
\text { value }\end{array}$ & $\begin{array}{c}\text { Biomass } \\
\text { (dry yeast g / })\end{array}$ & $\begin{array}{c}\text { Astaxanthin } \\
(\mathrm{mg} / \mathrm{g} \text { yeast })\end{array}$ & $\begin{array}{c}\text { Astaxanthin } \\
\text { productivity } \\
(\mathrm{mg} / \mathrm{l})\end{array}$ \\
\hline 4.0 & $0.042 \pm 0.015$ & $\mathrm{~N}^{\mathrm{D}} .^{\text {a }}$ & N.D. $^{\text {a }}$ \\
5.0 & $1.570 \pm 0.045$ & $0.8817 \pm 0.080$ & $1.3842 \pm 0.099$ \\
$5.2^{\mathrm{b}}$ & $2.070 \pm 0.123$ & $0.8527 \pm 0.064$ & $1.7650 \pm 0.097$ \\
6.0 & $2.000 \pm 0.126$ & $0.7243 \pm 0.078$ & $1.4486 \pm 0.110$ \\
7.0 & $1.480 \pm 0.129$ & $0.5007 \pm 0.045$ & $0.7410 \pm 0.080$ \\
8.0 & $1.860 \pm 0.133$ & $0.2772 \pm 0.047$ & $0.5155 \pm 0.093$ \\
\hline
\end{tabular}

The results were presented as mean values \pm S.E. of three replicates

${ }^{a}$ N.D.: not detected.

${ }^{\mathrm{b}}$ Natural initial $\mathrm{pH}$ value. 
the wild-type $X$. dendrorhous (ATCC 24228) growing was $18-26^{\circ} \mathrm{C}$, and its optimal culture temperature was $21^{\circ} \mathrm{C}$, both biomass and astaxanthin could gain high production under this condition. While temperature excessed $26^{\circ} \mathrm{C}, \mathrm{X}$. dendrorhous almost could not grow. Table 2 showed that suitable initial $\mathrm{pH}$ value of $X$. dendrorhous culturing was 5.0-6.0, optimal initial $\mathrm{pH}$ value was natural $\mathrm{pH}$ value.

\section{Effect of nutritional factors on astaxanthin productivity}

Various nutritional media were utilized to optimize medium for astaxanthin-production yeast. Several media (Table 3) were compared under uniform physical conditions. The results (Table 3) indicated that 323malt, PDB and YM medium were good nutritional media for $X$. dendrorhous ATCC 24228 growth and astaxanthin production.

The property of Phaffia yeasts for growing on a variety of carbon sources, such as glucose, cellobiose, maltose, sucrose, lactose, xylose and arabinose [6,8,13], was a remarkable advantage.

To examine the effect of carbon sources on the astaxanthin production, flask cultures were performed by employing several carbon sources (Table 4). Glycerol and sucrose gave higher growth than glucose did, but their astaxanthin contents were far less than that on glucose. Lactose and galactose gave lower growth and higher astaxanthin content to glucose, while cellobiose gave a relative lower growth but higher astaxanthin content than glucose did. These results showed that glycerol and sucrose could greatly increase the biomass but greatly decrease astaxanthin content; glucose, galactose and cellobiose can gain relatively higher biomass and higher astaxanthin content than others, therefore gaining higher astaxanthin productivity.

In view of this fact that carotenogenesis in wild-type $X$. dendrorhous occurs after glucose was exhausted from the medium and high concentrations of glucose in batch culture decrease carotenogenesis and lead to accumulation of carotenes [9], the effect of various concentration glucose on astaxanthin productivity was investigated on base of medium PDB and YM. Results were listed in Table 5. Our results showed that higher concentration glucose could not greatly increase both biomass and astaxanthin content, while relatively low concentration glucose $(20-30 \mathrm{~g} / \mathrm{l})$

Table 3. Screening for optimal nutritional medium

\begin{tabular}{lcccc}
\hline Medium ID & $\begin{array}{c}\text { Biomass } \\
(\text { dry yeast g } / \mathrm{l})\end{array}$ & $\begin{array}{c}\text { Astaxanthin } \\
(\mathrm{mg} / \mathrm{g} \text { yeast })\end{array}$ & $\begin{array}{c}\text { Astaxanthin productivity } \\
(\mathrm{mg} / \mathrm{l})\end{array}$ & $\begin{array}{c}\text { Total carotenoids } \\
(\mathrm{mg} / \mathrm{g} \text { yeast })\end{array}$ \\
\hline 323malt & $2.262 \pm 0.229$ & $1.874 \pm 0.109$ & $4.241 \pm 0.201$ & $3.864 \pm 0.088$ \\
PDB & $2.166 \pm 0.099$ & $0.876 \pm 0.045$ & $1.898 \pm 0.082$ & $2.964 \pm 0.149$ \\
PDBY & $2.534 \pm 1.332$ & $0.284 \pm 0.089$ & $0.720 \pm 0.097$ & $1.190 \pm 0.113$ \\
YEPD & $1.942 \pm 0.210$ & $0.070 \pm 0.029$ & $0.135 \pm 0.091$ & $0.520 \pm 0.142$ \\
YM & $2.625 \pm 0.133$ & $1.043 \pm 0.229$ & $2.737 \pm 0.156$ & $3.203 \pm 0.329$ \\
NA & $0.730 \pm 0.118$ & $0.014 \pm 0.009$ & $0.010 \pm 0.005$ & $0.044 \pm 0.014$ \\
\hline
\end{tabular}

The results were presented as mean values \pm S.E. of three replicates

Table 4. Effect of carbon sources on biomass and astaxanthin yield on PDB medium

\begin{tabular}{lcccc}
\hline Carbon source & $\begin{array}{c}\text { Biomass } \\
(\text { dry yeast g/l) })\end{array}$ & $\begin{array}{c}\text { Astaxanthin } \\
(\mathrm{mg} / \mathrm{g} \text { yeast })\end{array}$ & $\begin{array}{c}\text { Astaxanthin productivity } \\
(\mathrm{mg} / \mathrm{l})\end{array}$ & $\begin{array}{c}\text { Total carotenoids } \\
(\mathrm{mg} / \mathrm{g} \text { yeast })\end{array}$ \\
\hline Blank $^{\mathrm{a}}$ & $2.087 \pm 0.321$ & $0.849 \pm 0.078$ & $1.772 \pm 0.259$ & $2.773 \pm 0.321$ \\
Glucose $^{\mathrm{b}}$ & $3.870 \pm 0.522$ & $0.172 \pm 0.039$ & $0.665 \pm 0.133$ & $0.954 \pm 0.104$ \\
Fructose $^{\mathrm{b}}$ & $4.290 \pm 0.427$ & $0.216 \pm 0.079$ & $0.929 \pm 0.258$ & $0.741 \pm 0.149$ \\
Sucrose $^{\mathrm{b}}$ & $5.908 \pm 0.098$ & $0.021 \pm 0.013$ & $0.125 \pm 0.084$ & $0.441 \pm 0.033$ \\
Glycerol $^{\mathrm{b}}$ & $13.331 \pm 0.193$ & $0.035 \pm 0.017$ & $0.473 \pm 0.102$ & $0.608 \pm 0.155$ \\
Maltose $^{\mathrm{b}}$ & $3.320 \pm 0.288$ & $0.168 \pm 0.103$ & $0.558 \pm 0.123$ & $1.297 \pm 0.277$ \\
Cellobiose $^{\mathrm{b}}$ & $3.489 \pm 0.120$ & $0.501 \pm 0.215$ & $1.751 \pm 0.084$ & $1.244 \pm 0.235$ \\
Lactose $^{\mathrm{b}}$ & $1.320 \pm 0.192$ & $0.791 \pm 0.031$ & $1.044 \pm 0.082$ & $2.077 \pm 0.157$ \\
Galactose $^{\mathrm{b}}$ & $1.680 \pm 0.102$ & $0.979 \pm 0.219$ & $1.645 \pm 0.328$ & $1.323 \pm 0.202$ \\
\hline
\end{tabular}

The results were presented as mean values \pm S.E. of three replicates ${ }^{\mathrm{a}} \mathrm{PDB}$ containing glucose $20 \mathrm{~g} / 1$ and potato infusion solids $4 \mathrm{~g} / \mathrm{l}$.

${ }^{\mathrm{b}}$ Each carbon source $20 \mathrm{~g} / 1$ was added to PDB medium. 
Table 5. Effect of glucose concentration on astaxanthin productivity on base of medium PDB and YM

\begin{tabular}{cccccc}
\hline Medium ID & $\begin{array}{c}\text { Additive glucose } \\
(\mathrm{g} / \mathrm{l})\end{array}$ & $\begin{array}{c}\text { Biomass } \\
\text { (dry yeast } \mathrm{g} / \mathrm{l})\end{array}$ & $\begin{array}{c}\text { Astaxanthin } \\
(\mathrm{mg} / \mathrm{g} \text { yeast })\end{array}$ & $\begin{array}{c}\text { Astaxanthin productivity } \\
(\mathrm{mg} / \mathrm{l})\end{array}$ & $\begin{array}{c}\text { Total carotenoids } \\
\text { (mg/g yeast) }\end{array}$ \\
\hline PDB1 & 5 & $2.346 \pm 0.201$ & $0.744 \pm 0.037$ & $1.746 \pm 0.110$ & $1.494 \pm 0.407$ \\
PDB2 & 10 & $2.339 \pm 0.626$ & $0.865 \pm 0.143$ & $2.023 \pm 0.358$ & $2.773 \pm 0.390$ \\
PDB3 & 20 & $2.884 \pm 0.268$ & $0.495 \pm 0.091$ & $1.428 \pm 0.178$ & $2.065 \pm 0.356$ \\
PDB4 & 40 & $2.896 \pm 0.180$ & $0.517 \pm 0.083$ & $1.499 \pm 0.499$ & $2.300 \pm 0.578$ \\
PDB5 & 60 & $3.154 \pm 0.644$ & $0.471 \pm 0.105$ & $1.487 \pm 0.379$ & $2.044 \pm 0.369$ \\
PDB6 & 80 & $4.002 \pm 0.776$ & $0.503 \pm 0.087$ & $2.014 \pm 0.431$ & $2.199 \pm 0.394$ \\
\hline YM 1 & 5 & $2.777 \pm 0.589$ & $0.844 \pm 0.167$ & $2.344 \pm 0.321$ & $2.555 \pm 0.357$ \\
YM 2 & 10 & $2.840 \pm 0.444$ & $0.976 \pm 0.220$ & $2.772 \pm 0.313$ & $2.927 \pm 0.327$ \\
YM 3 & 20 & $2.954 \pm 0.863$ & $0.819 \pm 0.091$ & $2.422 \pm 0.390$ & $2.828 \pm 0.255$ \\
YM 4 & 40 & $2.977 \pm 0.663$ & $0.843 \pm 0.237$ & $2.513 \pm 0.326$ & $2.333 \pm 0.396$ \\
YM 5 & 60 & $3.222 \pm 0.306$ & $0.703 \pm 0.111$ & $2.266 \pm 0.225$ & $2.022 \pm 0.319$ \\
YM 6 & 80 & $2.087 \pm 0.295$ & $0.725 \pm 0.121$ & $1.514 \pm 0.227$ & $2.110 \pm 0.339$ \\
\hline
\end{tabular}

The results were presented as mean values \pm S.E. of three replicates

gained high astaxanthin and carotenoids content, these results also were proved by $\mathrm{Xu}$ et al. [17].

Since nitrogen significantly affected carotenogenesis $[3$, 16], the effect of various nitrogen sources on carotenogenesis and biomass production in $X$. dendrorhous was examined (Table 6). Sodium nitrate could increase both biomass and astaxanthin content on basal medium PDB, but did not significantly affect biomass and astaxanthin content in nutritional medium YM. The other nitrogen sources significantly decreased carotenogenesis both in PDB medium and YM medium.

\section{Effect of carotenogenesis stimulating factors on astaxanthin productivity}

Astaxanthin was formed via the mevalonate pathway, which starts at acetyl-CoA and proceeds via mevalonate to isopentenyl-pyrophosphate (IPP), the general precursor of all isoprenoids. Subsequently eight molecules of IPP were condensed to form the colorless carotenoid phytoene. Via four dehydrogenation and two cyclization reactions phytoene was converted into $\beta$-carotene. Finally $\beta$-carotene was oxidized to yield astaxanthin [4]. The existence of a monocyclic carotenoid-biosynthetic pathway in X. dendro-

Table 6. Effect of additive nitrogen source on astaxanthin productivity on base of medium PDB and YM

\begin{tabular}{|c|c|c|c|c|c|}
\hline Medium ID & Nitrogen Sources ${ }^{a}$ & $\begin{array}{c}\text { Biomass } \\
(\text { dry yeast } \mathrm{g} / \mathrm{l})\end{array}$ & $\begin{array}{l}\text { Astaxanthin } \\
\text { (mg/g yeast) }\end{array}$ & $\begin{array}{l}\text { Astaxanthin productivity } \\
(\mathrm{mg} / \mathrm{l})\end{array}$ & $\begin{array}{l}\text { Total carotenoids } \\
\text { (mg/g yeast) }\end{array}$ \\
\hline PDB & Blank & $2.770 \pm 0.313$ & $0.841 \pm 0.154$ & $2.329 \pm 0.269$ & $2.562 \pm 0.321$ \\
\hline PDBY & Yeast & $3.350 \pm 0.256$ & $0.209 \pm 0.113$ & $0.703 \pm 0.127$ & $1.047 \pm 0.269$ \\
\hline PDBS & $\left(\mathrm{NH}_{4}\right)_{2} \mathrm{SO}_{4}$ & $2.000 \pm 0.118$ & $0.043 \pm 0.021$ & $0.086 \pm 0.027$ & $0.125 \pm 0.135$ \\
\hline PDBU & Urea & $1.630 \pm 0.294$ & N.D. ${ }^{b}$ & 0.000 & $0.003 \pm 0.000$ \\
\hline PDBC & $\left(\mathrm{NH}_{4}\right)_{2}$ Citrate & $2.560 \pm 0.321$ & $0.382 \pm 0.034$ & $0.978 \pm 0.137$ & $1.045 \pm 0.148$ \\
\hline PDBN & $\mathrm{NaNO}_{3}$ & $3.612 \pm 0.032$ & $1.410 \pm 0.289$ & $5.090 \pm 0.365$ & $4.590 \pm 0.390$ \\
\hline PDBA & $\left(\mathrm{NH}_{4}\right)$ Acetate & $1.953 \pm 0.235$ & N.D. ${ }^{\mathrm{b}}$ & 0.000 & $0.097 \pm 0.043$ \\
\hline YM & Blank & $3.010 \pm 0.248$ & $0.834 \pm 0.120$ & $2.511 \pm 0.542$ & $2.845 \pm 0.290$ \\
\hline YMM & Malt. Ext & $3.070 \pm 0.477$ & $0.765 \pm 0.052$ & $2.350 \pm 0.302$ & $2.993 \pm 0.035$ \\
\hline YMS & $\left(\mathrm{NH}_{4}\right)_{2} \mathrm{SO}_{4}$ & $2.030 \pm 0.225$ & $0.153 \pm 0.033$ & $0.310 \pm 0.092$ & $1.007 \pm 0.174$ \\
\hline YMU & Urea & $1.440 \pm 0.118$ & N.D. ${ }^{\mathrm{b}}$ & 0.000 & $0.057 \pm 0.031$ \\
\hline YMC & $\left(\mathrm{NH}_{4}\right)_{2}$ Citrate & $1.442 \pm 0.321$ & $0.521 \pm 0.142$ & $0.752 \pm 0.124$ & $2.002 \pm 0.127$ \\
\hline YMN & $\mathrm{NaNO}_{3}$ & $2.090 \pm 0.543$ & $0.705 \pm 0.172$ & $1.473 \pm 0.323$ & $3.004 \pm 0.653$ \\
\hline YMA & $\left(\mathrm{NH}_{4}\right)$ Acetate & $2.040 \pm 0.393$ & $0.233 \pm 0.128$ & $0.475 \pm 0.235$ & $1.075 \pm 0.034$ \\
\hline
\end{tabular}

The results were presented as mean values \pm S.E. of three replicates

${ }^{a}$ Each nitrogen source was added at $0.2 \%(\mathrm{w} / \mathrm{w})$.

${ }^{\mathrm{b}}$ N.D.: not detected. 
Table 7. Effect of precursor additive composition on astaxanthin productivity

\begin{tabular}{|c|c|c|c|c|c|}
\hline Medium & Additive composition & $\begin{array}{c}\text { Biomass } \\
\text { (dry yeast g/l) }\end{array}$ & $\begin{array}{l}\text { Astaxanthin } \\
\text { (mg/g yeast) }\end{array}$ & $\begin{array}{l}\text { Astaxanthin productivity } \\
(\mathrm{mg} / \mathrm{l})\end{array}$ & $\begin{array}{c}\text { Total carotenoids } \\
\text { (mg/g yeast) }\end{array}$ \\
\hline \multirow{6}{*}{ 323malt } & Blank & $2.500 \pm 0.312$ & $1.563 \pm 0.214$ & $3.908 \pm 0.234$ & $3.225 \pm 0.218$ \\
\hline & Tomato $^{\mathrm{a}}$ & $3.000 \pm 0.092$ & $2.205 \pm 0.083$ & $6.617 \pm 0.031$ & $6.776 \pm 0.321$ \\
\hline & Carrot $^{\mathrm{b}}$ & $3.860 \pm 0.443$ & $2.521 \pm 0.236$ & $9.731 \pm 0.156$ & $10.42 \pm 10.632$ \\
\hline & Mevalonic acid & $4.600 \pm 0.542$ & $0.772 \pm 0.043$ & $3.553 \pm 0.442$ & $3.032 \pm 0.421$ \\
\hline & Acetic acid $^{\mathrm{d}}$ & $2.730 \pm 0.387$ & $1.224 \pm 0.395$ & $3.341 \pm 0.265$ & $4.389 \pm 0.762$ \\
\hline & Citric acid $^{\mathrm{e}}$ & $1.865 \pm 0.231$ & $0.235 \pm 0.239$ & $0.439 \pm 0.229$ & $0.896 \pm 0.321$ \\
\hline \multirow{6}{*}{ YM } & lank & $2.540 \pm 0.321$ & $0.901 \pm 0.044$ & $2.288 \pm 0.279$ & $3.044 \pm 0.683$ \\
\hline & Tomato $^{\mathrm{a}}$ & $3.920 \pm 0.265$ & $0.454 \pm 0.111$ & $1.780 \pm 0.173$ & $1.664 \pm 0.253$ \\
\hline & Carrot $^{\mathrm{b}}$ & $6.960 \pm 0.444$ & $2.038 \pm 0.287$ & $14.187 \pm 0.543$ & $8.003 \pm 0.321$ \\
\hline & Mevalonic acid & $6.000 \pm 0.245$ & $1.632 \pm 0.246$ & $9.792 \pm 0.312$ & $4.990 \pm 0.431$ \\
\hline & Acetic acid ${ }^{\mathrm{d}}$ & $6.200 \pm 0.321$ & $0.954 \pm 0.219$ & $5.916 \pm 0.431$ & $2.887 \pm 0.321$ \\
\hline & Citric acid $^{\mathrm{e}}$ & $4.550 \pm 0.573$ & $1.060 \pm 0.031$ & $4.825 \pm 0.321$ & $3.060 \pm 0.321$ \\
\hline
\end{tabular}

The results were presented as mean valuesS.E. of three replicates.

${ }^{a}$ Added $5 \mathrm{ml}$ tomato extract $(1 \mathrm{mg} / \mathrm{ml})$. ${ }^{\mathrm{b}}$ Added $5 \mathrm{ml}$ carrot extract $(1 \mathrm{mg} / \mathrm{ml}) . \quad{ }^{\mathrm{c}} 0.05 \mathrm{M}$ mevalonic acids.

${ }^{\mathrm{d}}$ Added $0.4 \%(\mathrm{~V} / \mathrm{V})$ acetic acid.

${ }^{\mathrm{e}}$ Added $12.5 \mathrm{uM}$ citric acid.

rhous was proposed by An et al. [2]. The monocyclic pathway diverged from the dicyclic pathway at neurosporene and proceeds through $\beta$-zeacarotene, $\gamma$-carotene, torulene, 3-hydroxy-3', 4'-didehydro- $\beta$, - $\Psi$-carotene-4-one (HDCO) to the end product 3,3'-dihydroxy- $\beta$, - $\Psi$-carotene4, 4'-dione.

The knowledge about the regulation of carotenogenesis in $X$. dendrorhous is still limited in comparison to that in other carotenogenic fungi. In many organisms, mevalonate synthesis, which was an early step in terpenoid biosynthesis, was a key point of regulation [7] of the corresponding pathway. This was probably also true for fungi, including $X$. dendrorhous. In fact, addition of mevalonate to a culture of $X$. dendrorhous stimulated both astaxanthin and total carotenoid biosynthesis four times [5].

To determine the effect of precursor substance of astaxanthin biosynthesis on growth and astaxanthin production by $X$. dendrorhous, two optimized nutritional medium 323malt and YM were supplemented with different precursor additive composition (Table 7), and the optimized culture conditions in our study were used for cultivation. The results showed that tomato extract, carrot extract, mevalonic acid and acetic acid could greatly increase astaxanthin productivity and carotenoids content in both medium 323malt and medium YM. Citric acid could greatly increase astaxanthin productivity in medium YM, but decreased astaxanthin productivity in medium 323malt.

According to carotenoid biosynthetic pathway theory proposed by Andrewes et al. [4], carotenoid biosynthesis come of mevalonate pathway, acetic acid and mevalonic acid as initial substance and intermediate substance of mevalonate pathway, played key role in the course of initial stage of carotenoid biosynthesis. Though mevalonate acid significantly increased astaxanthin production which was proved by Calo et al. [5] and us, mevalonate acid was too expensive to use in large-scale production of astaxanthin, however, as a cheap precursor composition, acetic acid can be applied to large scale production of astaxanthin. As we know, tomato extract rich with lycopene and carrot extract rich with beta-carotene, lycopene and beta-carotene as intermediate substance of astaxanthin biosynthesis also act as important role. Tomato extract and carrot extract provided both nutrition and precursor compositions of astaxanthin production, therefore improved production of astaxanthin. As cheap and easy available astaxanthin-genesis stimulating substance, tomato extract and carrot extract could increase both biomass and astaxanthin yield, so these substance could be apply to commercial production of astaxanthin.

\section{References}

1. An, G. H., C. H. Kim, E. S. Choi, and S. K. Rhee. 1996. Medium optimiza-tion of acarotenoid -hyperproducing Phaffia rhodozyma mutant HT-5FO1C. J. Ferment. Bioeng. 82, 515-518.

2. An, G. H., M. H. Cho, and E. A. Johnson. 1999. Monocyclic carotenoid biosynthetic pathway in the yeast Phaffia 
rhodozyma (Xanthophyllomyces dendrorhous). J. Biosci. Bioeng. 88, 189-193.

3. An, G. H. 2001. Improved growth of the red yeast Phaffia rhodozyma, in the presence of tricarboxylic acid cycle intermediates. Biotechnol. Lett. 23, 1005-1009.

4. Andrewes, A. G., H. J. Phaff, and M. P. Starr. 1976. Carotenoids of Phaffia rhodozyma, a red-pigmented fermenting yeast. Phytochemistry 15, 1003-1007.

5. Calo, P., T. D. Miguel, J. B. Velzquez, and T. G. Villa. 1995. Mevalonic acid increases trans-astaxanthin and carotenoid biosynthesis in Phaffia rhodozyma. Biotechnol. Lett. 17, 575-578.

6. Fang, T. J., and Y. S. Cheng. 1993. Improvement of astaxanthin production by a Phaffia rhodozyma through mutation and optimization of culture conditions. J. Ferment. Bioeng. 75, 466-469.

7. Goldstein, J. L. and M. S. Brown. 1990. Regulation of the mevalonate pathway. Nature 343, 425-430.

8. Johnson, E. A. and G. H. An. 1991. Astaxanthin from microbial sources. CRC Critical Reviews in Biotechnology 11, 297-326.

9. Johnson, E. A. and M. J. Lewis. 1979. Astaxanthin formation by the yeast Phaffia rhodozyma. J. Gen. Microbiol. 115, 173-183.

10. Meyer, P. S., and J. C. Du preez. 1994. Effect of culture conditions on astaxanthin production by a mutant of Phaffia rhodozyma in batch and chemostat culture. Appl. Microbiol. Biotechnol. 40, 780-785.

11. Miki, W. 1991. Biological functions and activities of animal carotenoids. Pure Appl. Chem. 63, 141-146.

12. Naguib, Y. 2000. Antioxidant activities of astaxanthin and related carotenoids. J. Agr. Chem. 48, 1150-1154.

13. Parajo, J. C., V. Santos, and M. Vazquez. 1997. Production of carotenoids by Xanthophyllomyces dendrorhous growing on enzymatic hydrolysates of pre-hydrolysed wood. Food chemistry 60, 347-355.

14. Schroeder, W. A., and E. A. Johnson. 1993. Antioxidant role of carotenoids in Phaffia rhodozyma. J. Gen. Microbiol. 139, 907-912.

15. Shinidzu, N., M. Goto, and W. Miki. 1996. Carotenoids as singlet oxygen quenchers in marine organisms. Fish. Sci. 61, 134-137.

16. Spencer, K. G. 1989. Pigmentation supplements for animal feed composition. US Patent 4,871,551.

$17 \mathrm{Xu}$, X. M., Z. Y. Jin, and D. H. Liu. 2000. Astaxanthin production by Phaffia rhodozyma in shake flask culture. Journal of Wuxi University of Light Industry (in Chinese) 19, 230-235.

\section{초록 : Carotenogenesis의 생성 자극 인자를 이용한 Xanthophyllomyces dendrorhous에 의한 astaxanthin 산물의 증가 \\ 서보군 ${ }^{1} \cdot$ 이장전 · 모은경 ${ }^{2} \cdot$ 성창근 \\ (충남대학교 농업생명과학대학 식품공학과, ${ }^{1}$ 중국 대련시 대련대학 약물연구소, ${ }^{2}$ (주)대덕바이오 생명과학연구소)}

야생 균주 Xanthophyllomyces dendrorhous으로부터 biomass와 astaxanthin 산물을 증가시키기 위해 shake flask 배양 조건에서 astaxanthin 산물에 영향을 미치는 물리적 인자, 영양 인자와 Carotenogenesis의 생성 자 극 인자에 대한 연구를 HPLC 분석 방법을 이용하여 수행하였다. 최적에 온도, 초기 $\mathrm{pH}$ 값, 탄소와 질소 공급 조건에서 Carotenogenesis의 선구 물질인 acetic 산, mevalonic 산, 토마토 추출물과 당근 추출물은 astaxanthin 생산을 상당히 증가시킬 수 있었다. 